\title{
Refractive Lens Exchange
}

\author{
Ahmed El-Massry, ${ }^{1}$ Eiman Abdellatif ${ }^{2}$ and Mohamed Ahmed El-Massry ${ }^{3}$ \\ 1. Professor of Ophthalmology; 2. Lecturer of Ophthamology; \\ 3. Third Year Residence and ICO Fellow, Department of Ophthalmology, Alexandria University, Egypt
}

\begin{abstract}
Refractive lens exchange (RLE) still has a role in young age with anisometropia, or in an eye that is anatomically not suitable for phakic intraocular lens (IOL) implantation or in high hypermetropia. Furthermore, in the presence of water vacuoles in the crystalline lens above the age of 40, it is recommended to perform RLE rather than phakic IOL implantation. Multifocal refractive IOLs are designed with several optical zones on the IOL. Apodised diffractive multifocal IOL has gradual diffractive steps on the IOL implant that create a smooth transition between focal points. RLE is a surgery with the same risks of a cataract surgery. Complications do occur in RLE. The mean incidence of retinal detachment is $1 \%$ while the mean incidence of cystoid macular oedema is $0.1 \%$. Contraindications include unstable refraction, amblyopia and the presence of certain eye pathologies as age-related macular degeneration, glaucoma, diabetic retinopathy and a high risk of retinal detachment.
\end{abstract}

\section{Keywords}

Refractive lens exchange, multifocal refractive IOLS

Disclosure: Ahmed El-Massry, Eiman Abdellatif and Mohamed Ahmed El-Massry have no conflicts of interest to declare. No funding was received in the publication of this article.

Open Access: This article is published under the Creative Commons Attribution Noncommercial License, which permits any non-commercial use, distribution, adaptation and reproduction provided the original author(s) and source are given appropriate credit.

Received: 15 June 2015 Accepted: 20 June 2015 Citation: European Ophthalmic Review, 2015;9(1):17-8 DOl: 10.17925/EOR.2015.09.01.17

Correspondence: Ahmed El-Massry, Professor of Ophthalmology, Ophthalmology Department, Alexandria University, Egypt. E: ahmad.elmassry@gmail.com

Emmetropia is defined as the state of refraction of the eye in which parallel rays of light falling on the cornea come to focus to a point on the retina while accommodation is at rest. Refractive errors comprising myopia, hyperopia and astigmatism, represent deviation from emmetropia, and if significant, they often require correction. ${ }^{1}$

There are various surgical options to correct refractive errors. The surgical armamentarium includes refractive corneal surgery, phakic intraocular lenses (IOLS) and refractive lens surgery. When laser refractive surgery is not a suitable approach, whether due to an excessively thin cornea or a too steep or too flat cornea, or a refractive error that is beyond what could be corrected with the available corneal thickness, we can resort to either phakic IOL implantation or refractive lens exchange (RLE). ${ }^{2}$

\section{Indications}

RLE still has a role in young age with anisometropia, or in an eye that is anatomically not suitable for phakic IOL implantation or in high hypermetropia. Furthermore, in the presence of water vacuoles in the crystalline lens above the age of 40 , it is recommended to perform RLE rather than phakic IOL implantation. ${ }^{2}$

In order to be compared to refractive surgeries, RLE should aim to cover the visual quality and accommodation, should induce minimal aberrations, should be as safe as laser refractive surgery and its cost should not be higher than laser refractive surgery.

Several methods are available for correction of presbyopia, including glasses (bifocal or multifocal), contact lenses (monovision or multifocal contact lenses), scleral surgeries (e.g. scleral expansion bands and anterior ciliarysclerotomy), laser-assisted in situ keratomileusis [LASIK] and presbyopia correcting IOLS). Presbyopia correcting IOLS include single optic IOLs, dual optic IOLs and deformable optic IOLS. New technology IOLs include Calhoun light adjustable IOLs, LiquiLens IOLs and the new bioptics (refractive IOLS + intracorneal lenses). ${ }^{3}$

\section{Intraocular Lenses}

Multifocal refractive IOLs are designed with several optical zones on the IOL. These zones provide multiple focal points, allowing for an improvement in distance, intermediate and near vision. The Rezoom ${ }^{\mathrm{TM}}$ multifocal lens has a patented new design called Balanced View optics technology. This lens design creates multiple focal points so the patient can see well at a variety of distances, be it near, middistance or far. ${ }^{3}$

On the other hand, the Acrysof ReSTOR IOL is an apodised diffractive multifocal IOL. This type of IOL has gradual diffractive steps on the IOL implant that create a smooth transition between focal points. The IOL also bends incoming light to the multiple focal points to increase vision in various lighting situations. This unique technology optimally manages light energy delivered to the retina because it distributes the appropriate amount of light to near and distant focal points, regardless of the lighting situation.

Apodised diffractive optics are designed to improve image quality while minimising visual disturbances. This results in an increased range of quality vision that delivers a high level of spectacle freedom. ${ }^{4}$ 
The SmartLens IOL is an injectable accommodating IOL design. It is made from a thermodynamic hydrophobic acrylic gel polymer and is flexible, and it can be made into any size, shape and diopteric power customised for the individual patient. At room temperature, the material can be formed into a rod of $3 \mathrm{~mm}$ in length and approximately $2 \mathrm{~mm}$ in width and can therefore be implanted into a patient's eye through a normal incision. Upon reaching body temperature, the IOL then reconfigurates into its original size, shape and diopteric power, filling the entire capsular bag.

The Acrysof Toric IOL is a single piece hydrophobic acrylic posterior toric IOL that corrects $1.03,1.55$ and 2.06 diopters (D) at the corneal plane. However for toric IOLS, for every 1 degree of rotation, $3.3 \%$ of the IOL cylinder power is lost. So 30 degrees of rotation may completely negate the cylinder power correction of a toric IOL.

\section{Complications}

RLE is a surgery with similar cataract surgery risks. It also presents the difficulties of IOL choice, the predictability of post-operative refraction, induced and residual astigmatism, retinal detachment, contrast sensitivity and the incidence of posterior capsule opacification (PCO). ${ }^{5}$

Regarding the visual results in myopic RLE, Colin et al. ${ }^{1}$ reported $100 \%$ improvement in uncorrected visual acuity (UCVA), $75 \%$ improvement in best-corrected visual acuity (BCVA) of 2 lines or more and $0-4 \%$ loss of BCVA. Gabric et al. ${ }^{6}$ found that RLE was predictable in $87.5 \%$ of cases within $1 \mathrm{D}$ and in $95.8 \%$ of cases within $2 \mathrm{D}$.

In hyperopic RLE, $88 \%$ of low hyperopes were within 1D of the target refraction while only $58 \%$ were within 1D of the target refraction.

Complications do occur in RLE. The mean incidence of retinal detachment is $1 \%$ while the mean incidence of cystoid macular oedema is $0.1 \%$. The risk of retinal detachment following RLE increases in young age, male gender, myopic subjects, eyes with an axial length exceeding $27 \mathrm{~mm}$, posterior capsule rupture and post-operative Yttrium aluminium garnet (YAG) capsulotomy. In the latter procedure, precautions to decrease the risk of retinal detachment include using low power laser and aiming for a small-diameter posterior capsulotomy.

On the other hand, a different bundle of complications occurs in eyes with a short axial length. These include suprachoroidal haemorrhage and malignant glaucoma.
For the best results, adequate pre-operative care should be exercised. The pre-operative patient counselling should include explaining the limitations of the surgery including the matter of presbyopia, exploring the patient's expectations and explaining the possible need for a post-operative enhancement as well as the possible post-operative visual symptoms.

\section{Contraindications}

Patient selection is of paramount importance. Contraindications include unstable refraction, amblyopia and the presence of certain eye pathologies such as age-related macular degeneration, glaucoma, diabetic retinopathy and a high risk of retinal detachment. Good candidates are presbyopic hyperopes, as well as high and moderate myopes.

Since available IOLs are inferior to the crystalline lens, then in order to give the patient an acceptable distance and reading vision, we need to use either monovision, monofocal IOLs plus reading glasses or multifocal IOLs.

RLE is an elective surgery, performed on eyes with 20/20 BCVA, in a patient with high expectations, hence before performing RLE, the surgeon needs to be comfortable with the technique of cataract surgery, and should be able to obtain predictable post-operative refraction in routine cataract surgeries first. In this respect, some modern biometry formulae have added greatly to the predictability of refractive outcomes in eyes with a too short or too long axial length.?

Moreover, special care should be given to minimising the relatively high risk of PCO in these young subjects. A well-centred anterior continuous curvilinear capsulorrhexis (ACCC) that covers the edge of the optic 360 degrees is essential, together with removing the lens' epithelial cells meticulously at the conclusion of surgery. Square edge acrylic IOLs also have a role in decreasing the incidence of PCO.

\section{Conclusions}

In conclusion, advances in IOL and phaco technology have added to the safety and efficiency of cataract surgery and guarantee of refractive results and allowed the procedure to be used for RLE in patients not suffering from cataract. Yet, RLE is not for every patient considering refractive surgery. Attention to proper patient selection, intraoperative technique and post-operative management has resulted in excellent outcomes.
1. Colin J, Robinet A, Clear lensectomy and implantation of low power posterior chamber intraocular lens for the correction of high myopia, Ophthalmology, 1994;101:107-12.

2. Gris $\mathrm{O}$, Guell J, Manero F, et al., Clear lens extraction to correct high myopia, J Cataract Refract Surg, 1996;22:686-9.

3. Dick HB, Accommodative intraocular lenses: current status,
Curr Opin Ophthalmol, 2005;16:8-26.

Hoffman RS, Fine IH, Packer M, Refractive lens exchange as a refractive surgery modality, Curr Opin Ophthalmol, 2004:15:22-8.

5. Barraquer C, Cavelier C, Mejia L, Incidence of retinal detachment following clear-lens extraction in myopic patients, Arch Ophthalmol, 1994;112:336-9

6. Gabric N, Dekaris I, Karaman Z, Refractive lens exchange correction of high myopia, Eur J Ophthalmol, 2002;12:384-7.

7. Fine $\mathrm{IH}$, Hoffman RS, Packer M, Optimizing refractive lens exchange with bimanual microincision phacoemulsification, J Cataract Refract Surg, 2004;30:550-4. 